\title{
Profile of natural anticoagulant, coagulant factor and anti-phospholipid antibody in critically ill COVID-19 patients
}

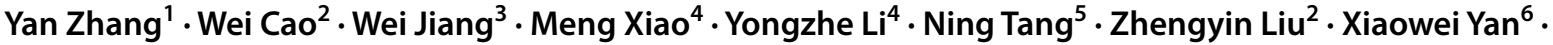 \\ Yongqiang Zhao ${ }^{1}$. Taisheng $\mathrm{Li}^{2}$. Tienan Zhu'
}

Published online: 9 July 2020

(c) Springer Science+Business Media, LLC, part of Springer Nature 2020

\begin{abstract}
The outbreak of novel coronavirus disease 2019 (COVID-19) has now become a global pandemic. Coagulopathy has been reported widely in critically ill COVID-19 patients and was related to high mortality. However, the comprehensive coagulation profiles have not been examined and the underlying mechanism of the coagulopathy in COVID-19 patients is unclear. To study the coagulation profiles of routine hemostasis tests, natural anticoagulants, coagulant factors and antiphospholipid antibodies in critically ill COVID-19 patients. This single-center and cross-section study included 19 patients with COVID-19, who were admitted to intensive care unit (ICU) at Tongji hospital in Wuhan, China, from Feb 23 to Mar 3, 2020. Demographic data, laboratory parameters, treatments and clinical outcomes of the patients were collected and analyzed. The final date of follow-up was Mar 31, 2020. In this study, 12 thrombotic events occurred in 9 patients, including 4 cerebral infarctions, 7 acro-ischemia and 1 internal jugular vein thrombosis. The common abnormalities of routine coagulation tests included evelated D-Dimer level (100\%), prolonged prothrombin time (73.7\%) and hyperfibrinogenemia (73.7\%). The median activities of natural anticoagulants including protein $\mathrm{C}$, protein $\mathrm{S}$ and antithrombin were all below the normal range. Factor VIII activities were significantly above normal range (median value 307\%, IQR 198-441) in all patients. Factor V and factor VII activities were significantly lower in near-terminal stage patients. Anti-phospholipid antibodies were present in 10 patients. Strikingly, 4 cerebral infarction events were in patients had anti-phospholipid antibodies of multiple isotypes. Sustained hypercoagulable status and thrombotic events were common in critically ill patients with COVID-19. The low activities of natural anticoagulants, elevated factor VIII level and the presence of antiphospholipid antibodies, together, may contribute to the etiopathology of coagulopathy in COVID-19 patients.
\end{abstract}

Keywords Critical · COVID-19 · Coagulopathy $\cdot$ Factor VIII $\cdot$ Antiphospholipid antibody

Taisheng Li

litsh@263.net

Tienan Zhu

zhutn@pumch.cn

1 Department of Hematology, Peking Union Medical College Hospital, Chinese Academy of Medical Sciences \& Peking Union Medical College, 1\# Shuai Fu Yuan, Beijing 100730, China

2 Department of Infectious Diseases, Peking Union Medical College Hospital, Chinese Academy of Medical Sciences \& Peking Union Medical College, 1\# Shuai Fu Yuan, Beijing 100730, China
3 Department of Medical ICU, Peking Union Medical College Hospital, Chinese Academy of Medical Sciences \& Peking Union Medical College, Beijing 100730, China

4 Department of Clinical Laboratory, Peking Union Medical College Hospital, Chinese Academy of Medical Sciences \& Peking Union Medical College, Beijing 100730, China

5 Department of Clinical Laboratory, Tongji Hospital, Tongji Medical College, Huazhong University of Science and Technology, Wuhan 430030, Hubei, China

6 Department of Cardiology, Peking Union Medical College Hospital, Chinese Academy of Medical Sciences \& Peking Union Medical College, Beijing 100730, China 


\section{Highlights}

- Hypercoagulable status is common in critically ill COVID-19 patients.

- The low activities of natural anticoagulants, elevated factor VIII level and the presence of antiphospholipid antibodies may contribute to the coagulopathy of COVID-19.

\section{Introduction}

Coronaviruses are important pathogens in humans that can cause diseases ranging from the common cold to more severe and even fatal respiratory infections. Since the outbreak of the novel coronavirus disease 2019 (COVID-19) caused by severe acute respiratory syndrome coronavirus 2 (SARS-CoV-2) in December 2019 in Wuhan, China, more than 2,000,000 cases have been diagnosed globally to date and the number is still growing rapidly. Patients infected with COVID-19 develop from mild, self-limiting respiratory tract illness to severe progressive pneumonia associated with multi-organ failure [1-4].

Recently, coagulopathy was reported widely present in severe SARS-CoV-2 infected patients and disseminated intravascular coagulation (DIC) developed in the majority of non-survivors [5]. The increase of D-dimer and clinical signs of organ damage indicating a significant hypercoagulable status were implicated in the formation of wide-spread microthrombi and multi-organ ischemia $[6,7]$. Although the pathological effect of SARS-CoV2 infection on coagulation system is unknown, release of various proinflammatory cytokines, vascular endothelial cell injuries, and platelet activation may play a role in the process. Indeed, data on coagulation activation in viral infection like coronaviruses are sparse. More recently, we reported the emergence of antiphospholipid antibodies (aPL) in 3 critically ill COVID-19 patients with multiple cerebral infarction [8]. The presence of these antibodies prompted us to further investigate the underlying mechanism of coagulopathy in COVID-19. Herein, we performed a cross-section study through analyzing coagulation profiles in 19 critically ill COVID-19 patients, to understand the coagulopathy of SASR-CoV2 infection, which may provide insights and guidance in clinical practice.

\section{Methods}

This single-center cross-sectional study included all critically ill patients who were admitted to the intensive care unit (ICU), Zhongfa campus of Tongji Hospital (Wuhan, China) between February 23 and March 3, 2020. The ICU was managed by the medical team from Peking Union Medical College Hospital (PUMCH). The patients were confirmed COVID-19 with oropharyngeal or nasopharyngeal swabs testing positive for SARS-CoV-2 by real-time reverse-transcription (PCR) using commercial kits (BGI BIOTECHNOLOGY (WUHAN) CO., LTD, Wuhan, China), according to the Chinese Recommendations for Diagnosis and Treatment of Novel Coronavirus (SARSCoV-2) Infection (Pilot 4th version) [9]. The treatments and outcomes (28-day mortality) were monitored up to March 30, 2020.

The study was approved by the Research Ethics Commission of Peking Union Medical College Hospital.

\section{Data collection}

Epidemiological, demographic, clinical, laboratory results, treatment, and outcome data were extracted from electronic medical records. Routine blood tests include complete blood count, coagulation profiles, serum biochemical tests (including renal and liver function, creatine kinase, lactate dehydrogenase, and electrolytes).

The coagulation profiles, including prothrombin time (PT), activated partial thromboplastin time (APTT), fibrinogen (FIB), D-dimer, fibrinogen degradation products (FDP) and antithrombin (AT) were detected using a STA-R MAX coagulation analyzer and manufacturersupplied reagents (or reagents supplied by the manufacturer) (Diagnostica Stago, Saint-Denis, France). Plasma activities of protein $\mathrm{C}$, protein $\mathrm{S}$, coagulation factor II, V, VII, VIII, IX, X, IX, XII were tested using STA-R MAX coagulation analyzer and manufacturer-supplied reagents (Diagnostica Stago, Saint-Denis, France) as well.

\section{Serum aPLs determination}

Serum anti-cardiolipin (aCL) antibodies (IgG, IgM and $\operatorname{Ig} \mathrm{A})$ and anti- $\beta 2$-glycoprotein 1 (aß2GP1) antibodies ( $\operatorname{IgG}, \operatorname{IgM}$, and $\operatorname{Ig} \mathrm{A}$ ) were determined by the chemiluminescence assay (CIA) (QUANTA Flash ${ }^{\circledR}$ assays, INOVA Diagnostic, Inc.) according to the manufacturer's instructions. The cutoff values for positivity were set $>20$ chemiluminescence units (CU) based on manufacturer's recommendations. 


\section{Lupus anticoagulant (LA)}

The detection of LA in human citrated plasma was performed by the HemosIL dRVVT Screen and HemosIL dRVVT Confirm assays, as recommended by the International Society on Thrombosis and Haemostasis (ISTH).

The onset of the disease was defined when the symptoms were noticed. Disseminated intravascular coagulation (DIC) was defined according to International Society of Thrombosis \& Hemostasis (ISTH) criteria of DIC [10]. Sepsis-induced coagulopathy (SIC) was defined according to the Third International Consensus Definitions for Sepsis (sepsis-3) [11]

\section{Statistical analysis}

Summary statistics were used to describe the study. Normal variables were summarized as means and standard deviations, and non-normal variables as medians and interquartile range (IQR). We used the $\chi^{2}$ test, Fisher's exact test or Mann-Whitney test to compare differences where appropriate. All statistical procedures were performed using SPSS 25.0 software (SPSS Inc., Chicago, IL, USA).

\section{Results}

\section{Clinical data}

A total of 20 patients were admitted to the ICU ward between February 23 and March 3, 2020 with confirmed
COVID-19 infection. Out of 20, 19 patients with integrated medical records were included in this study. One patient was excluded due to a lack of coagulation test results. All patients fulfilled the critically ill subtype according to the Diagnosis and Treatment Plan of COVID-19 suggested by National Health Commission of China [12].

The median age of the patients was 65.0 years (36-80). 14 patients (73.6\%) were older than 60 years. Sex distribution (F:M=9:10) was similar. Fourteen patients had underlying comorbidities, including hypertension, diabetes mellitus, stroke, chronic obstructive pulmonary disease, and malignancy. The median duration from onset to admission to ICU was 21 days (IQR 13-24.75). The treatments before ICU admission included: antiviral drugs (17/19), glucocorticoids (15/19), intravenous immunoglobulin (19/19), and antibiotics (18/19). All the patients received respiratory support after admission to ICU, 17(89.4\%) patients required endotracheal intubation and mechanical ventilation. The other supportive treatments included fluid management, nutritional support, antibiotics, continuous renal replacement therapy (2 patients) and extracorporeal membrane oxygenation (ECMO, for 1 patient). Due to the significant hypercoagulable status, 17 patients received low molecular weight heparin (LMWH) 4000-6000 IU, subcutaneous injection, twice per day. The 28-day mortality in ICU was $68.42 \%$, and the median duration in ICU of non-survivors was 14 (IQR 9-14) days (Table 1).

Thrombosis events occurred in 9 patients $(47.4 \%)$, including 7 acro-ischemia events, 4 cerebral infarction events and 1 jugular thrombosis event, as we reported previously $[6,8]$. The median interval from disease onset to coagulation profile sampling was 30 days (IQR 29-32).

Table 1 Clinical characters of critically ill patients with COVID-19

\begin{tabular}{llll}
\hline & Terminal stage $(\mathrm{N}=5)$ & Non-terminal $(\mathrm{N}=14)$ & Total $(\mathrm{N}=19)$ \\
\hline Age (median) & $71(63-72)$ & $65(59-68)$ & $65(60-70)$ \\
Male (n, \%) & $3(60 \%)$ & $7(50 \%)$ & $10(52.6 \%)$ \\
Comorbidity (n, \%) & $4(80 \%)$ & $8(57.2 \%)$ & $12(63.2 \%)$ \\
The interval from Onset to sampling (days, IQR) & $29(23-30)$ & $30(20-33.5)$ & $30(29-32)$ \\
Survival time (days, IQR) & $30(24-31)$ & $50(39.25-64.75)$ & $40(32-62)$ \\
SIC scores at sampling (IQR) & $5(4-6)$ & $2.5(2-4)$ & $3(2-5)$ \\
SOFA scores at sampling (IQR) & $13(11.5-15.5)$ & $7.5(5.5-10.25)$ & $9(6-13)$ \\
Overt-DIC scores at sampling (IQR) & $4(3-4)$ & $2(2-3)$ & $3(2-3.5)$ \\
Thrombotic events & 0 & 12 & 12 \\
Arterial thrombosis & 0 & 4 & 4 \\
Venous thrombosis & 0 & 1 & 7 \\
Micro-thrombi (acro-ischemia) & 0 & 7 & $1^{\mathrm{a}}$ \\
Bleeding events & $1^{\mathrm{a}}$ & 0 & 1 \\
\hline
\end{tabular}

DIC disseminated intravascular coagulation, SOFA sequential organ failure assessment, SIC sepsis-induced coagulopathy

a only 1 gastrointestinal bleeding event occurred after anticoagulation 


\section{Coagulation profiles}

All patients had significantly higher plasma D-dimer level $(3.24 \pm 1.98 \mu \mathrm{g} / \mathrm{ml}$, normal range $<0.5 \mu \mathrm{g} / \mathrm{ml})$, and FVIII activities (329.95 $\pm 141.80 \%$, normal range $60-150 \%$ ); High fibrinogen level and prolonged PT were seen in 14 patients. 11 patients had prolonged APTT and 7 patients developed thrombocytopenia.

We divided the patients into two groups: 5 patients who died within $24 \mathrm{~h}$ after the coagulation profile sampling was defined as the terminal-stage group; the other 14 patients lived more than 3 days since the coagulation profile sampling belonged to non-terminal stage group. The clinical characteristics and coagulation results were compared between the terminal-stage and the non-terminal-stage group. The SOFA score and SIC score were significantly higher (p 0.010) in the terminal-stage group and were consistent with clinical outcome. We also observed the significantly prolonged PT (17.2 s vs.15.2 s, p 0.044) and lower platelet count (88.0 vs $\left.214.0 \times 10^{9} / 1, \mathrm{p} 0.156\right)$ in the terminal-stage group. While the other routine coagulation parameters, including APTT,
FIB, D-dimer and FDP, were similar between the two groups (Table 2).

The levels of 3 natural anticoagulants were measured in this study. Protein $\mathrm{C}$ activities were below the normal range in both groups with the median activity of $63 \%$ (IQR 50-99). There was no significant difference between two groups $(66 \%$ v.s. $62 \%$, p 0.559). The finding in protein $\mathrm{S}$ and AT activities were similar (Table 2).

In terms of coagulant factors, factor VIII activity was significantly above the normal range in both groups, but without any difference (316\% v.s. $300 \%$, p 0.893). Factor II, V, VII, IX, X, XI, XII activities for both patients groups maintained in the normal range. However, factor V and factor VII activities were much lower in the terminal-stage group than the non-terminal-stage group, which was consistent with the prolonged PT in that group. (see Table 2)

\section{aPL profiles}

10 out of 19 patients (52.6\%) had positive serum aCL and/or a $\beta 2 \mathrm{GP} 1$ autoantibodies, and 7 out of these 10 patients had

Table 2 Coagulation parameter profile in critically ill patients with COVID-19

\begin{tabular}{|c|c|c|c|c|}
\hline & Terminal stage $(\mathrm{N}=5)$ & Non-termina $(\mathrm{N}=14)$ & Total $(\mathrm{N}=19)$ & $\mathrm{P}$ value \\
\hline PLT $\left(100-300 \times 10^{9} / 1\right)$ & $88(27.5-275)$ & $214(160.25-251.5)$ & $202(88-249)$ & 0.156 \\
\hline PT $(11.5-14.5 \mathrm{~s})$ & $17.7(16.35-19.6)$ & $15.2(14.125-16.4)$ & $15.8(14.5-17.7)$ & 0.044 \\
\hline FIB $(2.00-4.00 \mathrm{~g} / \mathrm{l})$ & $3.6(2.85-5.965)$ & $4.8(4.12-5.30)$ & $4.42(3.6-5.22)$ & 0.298 \\
\hline APTT $(29.0-42.0 \mathrm{~s})$ & $71.9(40.35-75.4)$ & $46.75(40.625-54.175)$ & $47.5(40.9-56.1)$ & 0.257 \\
\hline D-Dimer $(<0.5 \mu \mathrm{g} / \mathrm{ml}$ FEU $)$ & $3.61(0.81-6.835)$ & $2.72(1.995-4.105)$ & $2.72(1.55-4.93)$ & 0.754 \\
\hline FDP $(<5.0 \mu \mathrm{g} / \mathrm{ml})$ & $20.7(13.7-20.7)$ & $11.75(7.525-17.75)$ & $14.5(7.975-20.65)$ & 0.533 \\
\hline AT, $(80-120 \%)$ & $59(59-85)$ & $73(67-89.5)$ & $72.5(60.75-82.75)$ & 1.000 \\
\hline PC (70-142\%) & $66(26-88.5)$ & $62(56-106.5)$ & $63(50-99)$ & 0.559 \\
\hline PS (77-143\%) & $81(13-121)$ & $67.5(39.25-83.5)$ & $70(31-88)$ & 0.893 \\
\hline FVIII (60-150\%) & $316(184.5-488.5)$ & $300(216.5-414)$ & $307(198-441)$ & 0.893 \\
\hline FIX $(60-150 \%)$ & $137(76-169.5)$ & $143(115.75-182.75)$ & $137(119-176)$ & 0.500 \\
\hline FXI $(60-150 \%)$ & $54(29.5-164.5)$ & $98.5(32.5-97.25)$ & $92(43-138)$ & 0.500 \\
\hline FXII $(50-150 \%)$ & $45(33-85)$ & $56.5(32.5-97.25)$ & $56(43-87)$ & 0.687 \\
\hline FII (70-120\%) & $60(26.5-69)$ & $64(52.5-72.25)$ & $62(45-72)$ & 0.391 \\
\hline FV (70-120\%) & $68(50-106.5)$ & $160(100-193.25)$ & $129(73-190)$ & 0.005 \\
\hline FVII (55-170\%) & $58(31-72.5)$ & $98.5(77.25-107.25)$ & $93(58-106)$ & 0.014 \\
\hline FX $(70-120 \%)$ & $51(29-84.5)$ & $74(47-89.25)$ & $73(47-85)$ & 0.257 \\
\hline
\end{tabular}

All the parameters in this table were displayed by the form: median value, $25 \%$ to $75 \%$ IQR

$a C L$ anti-cardiolipin, $a \beta 2 G P 1$ anti- $\beta 2$-glycoprotein $1, A P T T$ activated partial thromboplastin time, $A T$ antithrombin, $F I B$ fibrinogen, $F D P$ fibrinogen degradation products, $L A$ lupus anticoagulant, $P C$ protein $\mathrm{C}, P L T$ platelet, $P T$ prothrombin time, $P S$ protein $\mathrm{S}$

Table 3 Antiphospholipid antibodies isotypes of critically ill patients with COVID-19

\begin{tabular}{|c|c|c|c|c|c|c|c|}
\hline & \multicolumn{3}{|l|}{$\mathrm{aCL}$} & \multicolumn{3}{|c|}{$\mathrm{a} \beta 2 \mathrm{GP} 1$} & \multirow[t]{2}{*}{ LA } \\
\hline & $\operatorname{IgA}$ & $\mathrm{IgG}$ & $\operatorname{IgM}$ & $\operatorname{IgA}$ & $\mathrm{IgG}$ & IgM & \\
\hline Positive & 6 & 2 & 1 & 7 & 6 & 0 & 1 \\
\hline
\end{tabular}

$a C L$ anti-cardiolipin, $a \beta 2 G P 1$ anti- $\beta 2$-glycoprotein $1, L A$ lupus anticoagulant 
multiple isotypes of aPLs (see Table 3). All 4 patients who developed cerebral infarction during the hospitalization had aPLs with multiple isotypes. Interestingly, no thrombotic events occurred in 9 aPL negative patients. Moreover, the patients positive aPLs had a lower 28-day mortality compared to those with negative aPL ( $40.0 \%$ vs $88.9 \%$, odd ratio $0.074,95 \%$ CI $0.139-0.871, \mathrm{p} 0.057)$. Only 1 patient in terminal-stage group had positive LA accompanied with high level of multiple aPLs (IgA aCL > 352.0 CU, IgA a $\beta 2 \mathrm{GP} 1$ 396.7 CU, IgG aCL 20.2 CU, IgG aß2GP1 45.5 CU).

\section{Discussion}

In the last two decades, two beta coronavirus epidemics have been identified as the cause of acute severe respiratory disease: Severe Acute Respiratory Syndrome (SARS) in 2003 and Middle East Respiratory Syndrome (MERS) in 2012. The coagulopathy of SARS included isolated transient elevations of the activated PTT in the first 2 weeks of infection in $63 \%$ patients, but most patients had normal prothrombin times and no elevation of D-dimers. A total of $2.5 \%$ of SARS patients showed evidence of DIC [13]. There has been no report about the incidence of coagulopathy in MERS. On the other hand, several studies have now demonstrated coagulopathy in SARS-Cov-2 infection since epidemic COVID-19 outbreak. However, none of the studies provided comprehensive coagulation evaluations $[5,14,15]$. To the best of our knowledge, this is the first study to investigate natural anticoagulants, coagulation factors combined with aPLs in critical COVID-19 patients.

Our study revealed significant aberrant coagulation changes of critically ill COVID-19 patients admitted to ICU in several aspects: fibrinolytic degradation products (D-dimer \& FDP) were significant above normal range in almost all patients; the levels of FIB and FVIII:C were above normal range in the majority of patients; the natural anticoagulant activities were mildly lower. Nearly half of patients developed thrombotic events after admission to ICU. These findings implied that sustained hypercoagulable status and activation of coagulation system are hallmarks of critically ill COVID-19, and provided a strong evidence to support anticoagulation therapy in these patients. These results are consistent with those from recent studies. Tang reported the elevated D-Dimer level in the inpatients with COVID-19 was related with higher mortality [5]. A relative large scaled study from Netherlands showed the incidence of symptomatic thrombotic event was 31\% in COVID-19 patients admitted to the ICU [16]. Another retrospective study in France reported the overall rate of VTE in anticoagulated patients was as high as $69 \%$ after using a systematic VTE screening strategy [17].
Different from prior studies, we not only tested the conventional coagulation parameters but also performed a comprehensive observation of coagulation system and natural anticoagulation system. Plasma levels of natural anticoagulants tend to be markedly reduced in patients with severe DIC [18]. In patients with sepsis, multiple trauma and major surgery, a correlation between the reduction in AT and disease severity has been shown [19]. AT and PC levels were related with hypercoagulable status and decreased levels develop earlier than overt DIC. Thus, AT and PC levels were considered diagnostic parameter for non-overt DIC in ISTH criteria [10]. In this study, the median values of AT and PC activity were below lower limit of normal. These results suggest that SARS-Cov-2 infection can induce similar hemostatic change just like severe bacterial pneumonia or sepsis in association with the development of a large amount of micro-thrombi formation and the consumption of the natural anticoagulants.

High plasma levels of proinflammatory cytokines (interleukin-2, interleukin-6, tumor necrosis factor- $\alpha$ and so on) have been observed in SARS-Con-2 patients admitted to ICU, suggesting that a cytokine storm effect developing in these individuals with severe disease aggravating the coagulopathy [20]. The significant elevated factor VIII activity and the high frequency of aPLs give us a clue for the explanation of sustained hypercoagulable state. This study showed that factor VIII activities were elevated in all patients. Factor VIII is a natural coagulant factor secreted by endothelial cells. Elevated plasma factor VIII coagulant activity (VIII:C) is widely accepted as an independent markers of increased thrombosis risk. In a population-based case control study, patients with factor VIII:C levels greater than $150 \%$ had an adjusted odds of 4.8 for thrombosis [21, 22]. The significantly elevated FVIII activity may be related with proinflammatory cytokines and contributes to the hypercoagulable status of COVID-19 patients.

Our preliminary data showed antiphospholid antibodies(aPLs) may be implicated in the exacerbation of hypercoagulable status. Most strikingly, all symptomatic thrombotic events occurred in aPL positive patients. IgA $\mathrm{aCL}, \operatorname{Ig} \mathrm{A} a \beta 2 \mathrm{GP} 1$ and $\mathrm{IgG}$ a $\beta 2 \mathrm{GP} 1$ were the most common antibodies in our study. This is different from immunodeficiency virus (HIV) and hepatitis $\mathrm{C}$ virus (HCV) infections that mainly induces IgG aCL antibodies [23]. IgA aß2GPI has been described as an independent risk factor for acute myocardial infarction and acute cerebral ischemia [24]. In this study, 4 patients who suffered cerebral infarction all had multiple aPLs including IgA aCL and a $32 \mathrm{GP} 1$. Secondary antiphospholipid syndrome (APS) should be considered in COVID-19 patients with positive aPLs. Dynamic monitoring of aPLs levels may be useful to determine the persistence of aPLs and to confirm the diagnosis of APS. 
In our study, longer PT and lower FVII and FV activities were observed in the terminal-stage group. Moreover, higher SIC and ISTH DIC scores were identified in those near-death patients. COVID-19 can lead to systemic injuries by attacking angiotensin-converting enzyme 2 (ACE2) expressed vascular endothelial cells. Endothelial cell damage and cytokines (especially interleukin 6) strongly induce the expression of tissue factor (TF) and subsequent activation of coagulation. The more obvious coagulation abnormalities may reflect that there were much severe and irreversible vascular endothelial cell injuries in the terminal-stage group. As a result, anticoagulation therapy may not be enough to stop the continuous activation of coagulation system in those patients and these patients may benefit from the addition of anti-cytokine storm treatment (such as tocilizumab).

This study had some limitations. First, this is a smallscale cross-section study. For practical reasons, we only repeated the conventional coagulation tests in our clinical practices with no dynamic surveillance of anticoagulants and coagulation factors. Second, only parts of coagulation and fibrinolysis parameters were tested. At last, due to the disease severity, head CT scan and deep vein thrombosis screen by ultrasound were performed in only symptomatic patients. If we had performed a systemic screening strategy, the incidence of thrombotic events would probably be even higher.

In conclusion, our current study demonstrated that the coagulopathy in critically ill COVID-19 patients was characterized by sustained hypercoagulable status and a high incidence of thrombotic events. The elevated FVIII:C activity combined with positive aPLs may be implicated in the mechanism of hypercoagulable status. Furthermore, the preliminary data showed a lower FV, FVII, and higher PT in terminal as compared to non-terminal COVID patients and may be valuable markers of advancing illness.

Acknowledgements The authors would like to acknowledge all the colleagues of PUMCH national medical team. We thank Jun Li, MD. $\& \mathrm{Ph} . \mathrm{D}$., for her great help in medical academic writing of English. We also thank Man-Chiu Poon, University of Calgary Cumming School of Medicine, Calgary, Alberta, Canada, for his critical revision of the manuscript.

Author contributions TNZ and TSL designed the study; YZ, WC, WJ performed the clinical data acquisition; MX carried on the laboratory test of antiphospholipid antibody; YZ contributed to statistical analysis and wrote the manuscript; NT managed the coagulation profile tests. TNZ, YZL revised the manuscript for important intellectual content critically. ZYL, XWY and TSL supervised the study.

\section{Compliance with ethical standards}

Conflict of interest All authors declare no competing interests.

\section{References}

1. Zhou F, Yu T, Du R, Fan G, Liu Y, Liu Z, Xiang J, Wang Y, Song B, Gu X, Guan L, Wei Y, Li H, Wu X, Xu J, Tu S, Zhang Y, Chen H, Cao B (2020) Clinical course and risk factors for mortality of adult inpatients with COVID-19 in Wuhan, China: a retrospective cohort study. Lancet 6736:1-9. https://doi.org/10.1016/S0140 -6736(20)30566-3

2. Guan W-J, Ni Z-Y, Hu Y, Liang W-H, Ou C-Q, He J-X, Liu L, Shan H, Lei C-L, Hui DSC, Du B, Li L-J, Zeng G, Yuen K-Y, Chen R-C, Tang C-L, Wang T, Chen P-Y, Xiang J, Li S-Y, Wang J-L, Liang Z-J, Peng Y-X, Wei L, Liu Y, Hu Y-H, Peng P, Wang J-M, Liu J-Y, Chen Z, Li G, Zheng Z-J, Qiu S-Q, Luo J, Ye C-J, Zhu S-Y, Zhong N-S, China Medical Treatment Expert Group for Covid-19 (2020) Clinical characteristics of coronavirus disease 2019 in China. N Engl J Med 382(18):1708-1720. https://doi. org/10.1056/NEJMoa2002032

3. Grasselli G, Zangrillo A, Zanella A, Antonelli M, Cabrini L, Castelli A, Cereda D, Coluccello A, Foti G, Fumagalli R, Iotti G, Latronico N, Lorini L, Merler S, Natalini G, Piatti A, Ranieri MV, Scandroglio AM, Storti E, Cecconi M, Pesenti A, COVID19 Lombardy ICU Network (2020) Baseline characteristics and outcomes of 1591 patients infected with SARS-CoV-2 admitted to ICUs of the Lombardy Region, Italy. JAMA 323(16):1574-1581. https://doi.org/10.1001/jama.2020.5394

4. Wang D, Hu B, Hu C, Zhu F, Liu X, Zhang J, Wang B, Xiang H, Cheng Z, Xiong Y, Zhao Y, Li Y, Wang X, Peng Z (2020) Clinical characteristics of 138 hospitalized patients with 2019 novel Coronavirus-Infected Pneumonia in Wuhan, China. JAMA. https ://doi.org/10.1001/jama.2020.1585

5. Tang N, Li D, Wang X, Sun Z (2020) Abnormal coagulation parameters are associated with poor prognosis in patients with novel coronavirus pneumonia. J Thromb Haemost 18:844-847. https://doi.org/10.1111/jth.14768

6. Zhang Y, Cao W, Xiao M, Li YJ, Yang Y, Zhao J, Zhou X, Jiang W, Zhao YQ, Zhang SY, Li TS (2020) Clinical and coagulation characteristics of 7 patients with critical COVID-2019 pneumonia and acro-ischemia. Chin J Hematol 41:E006. https://doi. org/10.3760/cma.j.issn.0253-2727.2020.0006

7. Zhou F, Yu T, Du R, Fan G, Liu Y, Liu Z, Xiang J, Wang Y, Song B, Gu X, Guan L, Wei Y, Li H, Wu X, Xu J, Tu S, Zhang Y, Chen H, Cao B (2020) Clinical course and risk factors for mortality of adult inpatients with COVID-19 in Wuhan, China: a retrospective cohort study. Lancet. https://doi.org/10.1016/S0140 -6736(20)30566-3

8. Zhang Y, Xiao M, Zhang S, Xia P, Cao W, Jiang W, Chen H, Ding $X$, Zhao H, Zhang H, Wang C, Zhao J, Sun X, Tian R, Wu W, Wu D, Ma J, Chen Y, Zhang D, Xie J, Yan X, Zhou X, Liu Z, Wang J, Du B, Qin Y, Gao P, Qin X, Xu Y, Zhang W, Li T, Zhang F, Zhao Y, Li Y, Zhang S (2020) Coagulopathy and antiphospholipid antibodies in patients with Covid-19. N Engl J Med 382(17):e38. https://doi.org/10.1056/NEJMc2007575

9. National Health Commission of the People's Republic of China. Chinese Recommendations for Diagnosis and Treatment of Novel Coronavirus (SARS-CoV2) Infection (Pilot 4th version), (2020). https://www.gov.cn/zhengce/zhengceku/2020-01/28/5472673/files /0f96c10cc09d4d36a6f9a9f0b42d972b.pdf

10. Taylor FB, Toh CH, Hoots WK, Wada H, Levi M, Scientific Subcommittee on Disseminated Intravascular Coagulation (DIC) of the International Society on Thrombosis, and Haemostasis (ISTH) (2001) Towards definition, clinical and laboratory criteria, and a scoring system for disseminated intravascular coagulation. Thromb Haemost 86:1327-1330

11. Iba T, Di Nisio M, Levy JH, Kitamura N, Thachil J (2017) New criteria for sepsis-induced coagulopathy (SIC) following the 
revised sepsis definition: a retrospective analysis of a nationwide survey. BMJ Open 7:e017046. https://doi.org/10.1136/bmjop en-2017-017046

12. General Office of National Health Commission, Diagnostic and treatment protocol for Novel Coronavirus Pneumonia (Trial version 6) (2020). https://www.gov.cn/zhengce/zhengceku/202002/19/content_5480948.htm.

13. Wong RSM, Wu A, To KF, Lee N, Lam CWK, Wong CK, Chan PKS, Ng MHL, Yu LM, Hui DS, Tam JS, Cheng G, Sung JJY (2003) Haematological manifestations in patients with severe acute respiratory syndrome: retrospective analysis. BMJ 326:1358-1362. https://doi.org/10.1136/bmj.326.7403.1358

14. Yin S, Huang M, Li D, Tang N (2020) Difference of coagulation features between severe pneumonia induced by SARS-CoV2 and non-SARS-CoV2. J Thromb Thrombolysis. https://doi. org/10.1007/s11239-020-02105-8

15. Tang N, Bai H, Chen X, Gong J, Li D, Sun Z (2020) Anticoagulant treatment is associated with decreased mortality in severe coronavirus disease 2019 patients with coagulopathy. J Thromb Haemost 18:1094-1099. https://doi.org/10.1111/jth.14817

16. Klok FA, Kruip MJHA, van der Meer NJM, Arbous MS, Gommers DAMPJ, Kant KM, Kaptein FHJ, van Paassen J, Stals MAM, Huisman MV, Endeman H (2020) Incidence of thrombotic complications in critically ill ICU patients with COVID-19. Thromb Res. https://doi.org/10.1016/j.thromres.2020.04.013

17. Llitjos J-F, Leclerc M, Chochois C, Monsallier J-M, Ramakers M, Auvray M, Merouani K (2020) High incidence of venous thromboembolic events in anticoagulated severe COVID-19 patients. J Thromb Haemost. https://doi.org/10.1111/jth.14869

18. Fourrier F, Chopin C, Goudemand J, Hendrycx S, Caron C, Rime A, Marey A, Lestavel P (1992) Septic shock, multiple organ failure, and disseminated intravascular coagulation. Compared patterns of antithrombin III, protein $\mathrm{C}$, and protein $\mathrm{S}$ deficiencies. Chest 101:16-23. https://doi.org/10.1378/chest.101.3.816
19. Iba T, Kidokoro A, Yagi Y (1998) The role of the endothelium in changes in procoagulant activity in sepsis. J Am Coll Surg 187:321-329. https://doi.org/10.1016/s1072-7515(98)00177-x

20. Huang C, Wang Y, Li X, Ren L, Zhao J, Hu Y, Zhang L, Fan G, Xu J, Gu X, Cheng Z, Yu T, Xia J, Wei Y, Wu W, Xie X, Yin W, Li H, Liu M, Xiao Y, Gao H, Guo L, Xie J, Wang G, Jiang R, Gao Z, Jin Q, Wang J, Cao B (2020) Clinical features of patients infected with 2019 novel coronavirus in Wuhan, China. Lancet 395:497-506. https://doi.org/10.1016/S0140-6736(20)30183-5

21. Kraaijenhagen RA, Koopman MM, Reitsma PH, Prins MH, van den Ende A, Büller HR (2000) High plasma concentration of factor VIIIc is a major risk factor for venous thromboembolism. Thromb Haemost 83:5-9

22. Tsai AW, Cushman M, Rosamond WD, Heckbert SR, Tracy RP, Aleksic N, Folsom AR (2002) Coagulation factors, inflammation markers, and venous thromboembolism: the longitudinal investigation of thromboembolism etiology (LITE). Am J Med 113:636-642. https://doi.org/10.1016/s0002-9343(02)01345-1

23. Asherson RA, Cervera R (2003) Antiphospholipid antibodies and infections. Ann Rheum Dis 62:388-393. https://doi.org/10.1136/ ard.62.5.388

24. Andreoli L, Fredi M, Nalli C, Piantoni S, Reggia R, Dall'Ara F, Franceschini F, Tincani A (2013) Clinical significance of IgA anticardiolipin and IgA Anti- $\beta 2$ glycoprotein I antibodies. Curr Rheumatol Rep 15:343. https://doi.org/10.1007/s11926-013-0343-1

Publisher's Note Springer Nature remains neutral with regard to jurisdictional claims in published maps and institutional affiliations. 